\title{
STABILITY PROBLEM OF SOME NONLINEAR DIFFERENCE EQUATIONS
}

\section{ALAA E. HAMZA and M.A. EL-SAYED}

Department of Mathematics, Faculty of Science, Cairo University, Giza, 12211, Egypt.

(Received July 10, 1996)

ABSTRACT. In this paper, we investigate the asymptotic stability of the recursive sequence

$$
x_{n+1}=\frac{\alpha+\beta x_{n}^{2}}{1+\gamma x_{n-1}}, \quad n=0,1, \ldots
$$

and the existence of certain monotonic solutions of the equation

$$
x_{n+1}=x_{n}^{p} f\left(x_{n}, x_{n-1}, \ldots, x_{n-k}\right), \quad n=0,1, \ldots
$$

which includes as a special case the rational recursive sequence

$$
x_{n+1}=\frac{\beta x_{n}^{p}}{1+\sum_{\imath=1}^{k} \gamma_{2} x_{n-\imath}^{p-r}}
$$

where $\alpha \geq 0, \beta>0, \gamma>0, \gamma_{\imath} \geq 0, i=1,2, \ldots, k, \sum_{\imath=1}^{k} \gamma_{\imath}>0, p \in\{2,3, \ldots\}$ and $r \in\{1,2, \ldots, p-1\}$. The case when $r=0$ has been investigated by Camouzis et. al. [1], and for $r=0$ and $p=2$ by Camouzis et. al. [2].

KEY WORDS AND PHRASES: Difference Equations, Monotonic solutions, stability.

1991 AMS SUBJECT CLASSIFICATION CODES: 39A10.

\section{INTRODUCTION}

Many authors studied the asymptotic behaviour of the recursive sequence

$$
x_{n+1}=x_{n} f\left(x_{n}, x_{n-1}, \ldots, x_{n-k}\right), \quad n=0,1, \ldots
$$

which includes as a special case the rational recursive sequence

$$
x_{n+1}=\frac{a+b x_{n}}{1+\sum_{\imath=1}^{k} \gamma_{\imath} x_{n-1}}, \quad n=0,1, \ldots
$$

See Jaroma et. al. [3]. Also, there are many results about permanence, global attractivity and asymptotic stability of equation (1.2), see Camouzis et. al. [2], Kocic and Ladas [4-5] and Kocic et. al. [6]. The investigation of the behaviour of solutions of the equation

$$
x_{n+1}=x_{n}^{p} f\left(x_{n}, x_{n-1}, \ldots, x_{n-k}\right), \quad n=0,1, \ldots
$$


was suggested by Kocic and Ladas [5]. This equation includes as a special case the equation

$$
x_{n+1}=\frac{\alpha+\beta x_{n}^{p}}{1+\gamma x_{n-1}}, \quad n=0,1, \ldots
$$

Our aim in this paper is to study the asymptotic stability of the rational recursive sequence (1.4) when $p=2$, see section 2. On the basis of the results of section 2 we also investigate the behaviour of solutions of equation (1.3), in section 3 . We show that under certain conditions on $f$, there exists two solutions of (1.3) such that one tends to zero and the other tends to infinity. See theorem 3.1. We apply this theorem to equation (1.4) when $\alpha=0$.

2. THE RECURSIVE SEQUENCE $x_{n+1}=\left(\alpha+\beta x_{n}^{2}\right) /\left(1+\gamma x_{n-1}\right)$

In this section we study the asymptotic stability for the rational recursive sequence

$$
x_{n+1}=\frac{\alpha+\beta x_{n}^{2}}{1+\gamma x_{n-1}}, \quad n=0,1, \ldots
$$

where $\alpha \geq 0$ and $\beta, \gamma>0$.

The linearized equation associated with $(2.1)$ about $\bar{x}$ is

$$
y_{n+1}-\frac{2 \beta \bar{x}}{1+\gamma \bar{x}} y_{n}+\frac{\gamma \bar{x}}{1+\gamma \bar{x}} y_{n-1}=0, \quad n=0,1, \ldots
$$

The characteristic equation of (2.2) about $\bar{x}$ is

$$
\lambda^{2}-\frac{2 \beta \bar{x}}{1+\gamma \bar{x}} \lambda+\frac{\gamma \bar{x}}{1+\gamma \bar{x}}=0
$$

Equation (2.3) can be rewritten in the form

$$
(\lambda-l \theta)^{2}=l^{2} \theta^{2}-\theta
$$

where

$$
l=\beta / \gamma \text { and } \theta=\frac{\gamma \bar{x}}{1+\gamma \bar{x}}
$$

We summarize the results of this section in the following

\section{THEOREM 2.1}

(1) If $\beta>\gamma$ and $\alpha=0$, then equation (2.1) has two equilibria:

$$
\bar{x}_{1}=0, \quad \bar{x}_{2}=\frac{1}{\beta-\gamma}
$$

and $\bar{x}_{1}$ is asymptotically stable while $\bar{x}_{2}$ is unstable. Neither of them is a global attractor.

(2) If $\beta<\gamma$ and $\alpha=0$, then the unique equilibrium point $\bar{x}=0$ of equation (2.1) is globally asymptotically stable.

(3) If $\beta<\gamma$ and $\alpha>0$, then the unique positive equilibrium point

$$
\bar{x}=\frac{\sqrt{1+4 \alpha(\gamma-\beta)}-1}{2(\gamma-\beta)}
$$

of equation (2.1) is asymptotically stable. 
(4) If $\beta=\gamma$, equation (2.1) has the unique equilibrium point $\bar{x}=\alpha$ which is asymptotically stable.

(5) If $\beta>\gamma, \alpha>0$ and $1>4 \alpha(\beta-\gamma)$, then equation (2.1) has two positive equilibria

$$
\bar{x}_{1}=\frac{1-\sqrt{1-4 \alpha(\beta-\gamma)}}{2(\beta-\gamma)}
$$

which is asymptotically stable, and

$$
\bar{x}_{2}=\frac{1+\sqrt{1-4 \alpha(\beta-\gamma)}}{2(\beta-\gamma)}
$$

which is unstable. None of them is a global attractor.

(6) If $\beta>\gamma, \alpha>0$ and $1=4 \alpha(\beta-\gamma)$, then equation (2.1) has the unique equilibrium $\bar{x}=1 / 2(\beta-\gamma)$ which is neither stable nor a global attractor.

(7) Assume that $\beta>\gamma, \alpha>0$ and $1<4 \alpha(\beta-\gamma)$. If the initial conditions $\left\{x_{-1}, x_{0}\right\}$ are such that

$$
x_{0} \geq x_{-1} \text { and } x_{0} \geq \frac{1}{\beta-\gamma}
$$

then $\left\{x_{n}\right\}$ tends to infinity monotonically.

\section{PROOF.}

(1) Assume that $\beta>\gamma$ and $\alpha=0$. The characteristic equation of equation (2.2) about $\bar{x}_{1}=0$ is $\lambda^{2}=0$. Hence $\bar{x}_{1}$ is asymptotically stable, by Corollary 1.3.2 Kocic and Ladas [5] page 14. The characteristic equation of equation $(2.2)$ about $\bar{x}_{2}=1 /(\beta-\gamma)$ is

$$
\lambda^{2}-2 \lambda+\frac{\gamma}{\beta}=0
$$

which has two solutions $\lambda=1 \pm \sqrt{1-\gamma / \beta}$. Therefore, $\bar{x}_{2}$ is unstable. The nonattractivity of equilibria $\bar{x}_{1}$ and $\bar{x}_{2}$ follows directly from theorems 3.3 and 5.1 of Camouzis et. al. [1].

(2) Assume that $\beta<\gamma$ and $\alpha=0$. Let $\left\{x_{n}\right\}$ be a positive solution of equation (2.1). We have

$$
\frac{x_{n+1}}{x_{n}}=\frac{\beta x_{n}}{1+\gamma x_{n-1}}<\frac{\beta}{\gamma} \frac{x_{n}}{x_{n-1}}
$$

Hence $x_{n+1} / x_{n}<(\beta / \gamma)^{n+1}\left(x_{0} / x_{-1}\right) \forall n \in \mathbf{N}$. Since $\beta / \gamma<1$, then $(\beta / \gamma)^{n+1}\left(x_{0} / x_{-1}\right)<1 \forall n \geq n_{0}$ for some $n_{0} \in \mathbf{N}$. Therefore, $x_{n+1}<x_{n} \forall n \geq n_{0}$. This implies that $\lim _{n \rightarrow \infty} x_{n}=0$, i.e., $\bar{x}=0$ is globally asymptotically stable.

(3) Suppose that $\beta<\gamma$ and $\alpha>0$. We can see that $|\lambda|<1$ for every solution $\lambda$ of the characteristic equation (2.4), about

$$
\bar{x}=\frac{\sqrt{1+4 \alpha(\gamma-\beta)}-1}{2(\gamma-\beta)} .
$$

Indeed, we have the following two cases

First case: $l^{2} \theta^{2}-\theta<0$. In this case $\lambda=l \theta \pm i r$, where $r^{2}=\theta-l^{2} \theta^{2}$. Hence $|\lambda|^{2}=l^{2} \theta^{2}+r^{2}=\theta<1$.

Second case: $l^{2} \theta^{2}-\theta \geq 0$. In this case $\lambda=l \theta \pm \sqrt{l^{2} \theta^{2}-\theta}$. Hence, $|\lambda| \leq l \theta+\sqrt{l^{2} \theta^{2}-\theta}$. Since $l<1$, then $(2 l-1) \theta<1$. Hence $(1-l \theta)^{2}>l^{2} \theta^{2}-\theta$ whence $l \theta+\sqrt{l^{2} \theta^{2}-\theta}<1$. Therefore $|\lambda|<1$. In both cases $|\lambda|<1$ and thus $\bar{x}$ is asymptotically stable. 
(4) Assume that $\beta=\gamma$. For every solution $\lambda$ of the characteristic equation (2.4) about $\bar{x}=\alpha$, we have $|\lambda|^{2}=\gamma \alpha /(1+\gamma \alpha)<1$. Therefore, $\bar{x}$ is asymptotically stable.

(5) Suppose that $\beta>\gamma, \alpha>0$ and $1>4 \alpha(\beta-\gamma)$. The characteristic equation of (2.2) about

$$
\bar{x}_{1}=\frac{1-\sqrt{1-4 \alpha(\beta-\gamma)}}{2(\beta-\gamma)}
$$

is obtained by setting $\bar{x}=\bar{x}_{1}$ in equation (2.5). Since $\bar{x}_{1}<1 / 2(\beta-\gamma)<1 /(\beta-\gamma)$, then $l \theta<1$. We can see that $|\lambda|<1$ for every solution $\lambda$ of equation (2.4). Indeed, we have the following two cases

First case: $l^{2} \theta^{2}-\theta<0$. In this case $\lambda=l \theta \pm i r$, where $r^{2}=\theta-l^{2} \theta^{2}$. Hence $|\lambda|^{2}=l^{2} \theta^{2}+r^{2}=\theta<1$.

Second case: $l^{2} \theta^{2}-\theta \geq 0$. In this case $\lambda=l \theta \pm \sqrt{l^{2} \theta^{2}-\theta}$. Hence, $|\lambda| \leq l \theta+\sqrt{l^{2} \theta^{2}-\theta}$. Since $\bar{x}_{1}<1 / 2(\beta-\gamma)$, then $\gamma \bar{x}_{1} /\left(1+\gamma \bar{x}_{1}\right)<\gamma /(2 \beta-\gamma)$, i.e., $\theta<1 /(2 l-1)$. Hence $(1-l \theta)^{2}>l^{2} \theta^{2}-\theta$ whence $l \theta+\sqrt{l^{2} \theta^{2}-\theta}<1$. Therefore $|\lambda|<1$. In both cases $|\lambda|<1$ and thus $\bar{x}_{1}$ is asymptotically stable. In a similar manner, it can be shown that

$$
\bar{x}_{2}=\frac{1+\sqrt{1-4 \alpha(\beta-\gamma)}}{2(\beta-\gamma)}
$$

is unstable. To show the nonattractivity of $\bar{x}_{1}$ and $\bar{x}_{2}$, one chooses the initial conditions $\left\{x_{-1}, x_{0}\right\}$ such that

$$
x_{0} \geq x_{-1} \text { and } x_{0} \geq \max \left\{\frac{1}{\beta-\gamma}, \tilde{x}_{2}\right\}
$$

We show by induction that $\left\{x_{n}\right\}$ is increasing. Indeed, we have

$$
x_{n+1}>\frac{\beta x_{n}^{2}}{1+\gamma x_{n-1}}, \quad n=0,1, \ldots
$$

Then

$$
x_{1}>x_{0} \frac{\beta x_{0}}{1+\gamma x_{0}} \geq x_{0} .
$$

Assume that there exists $m_{0} \geq 0$ such that

$$
x_{n+1}>x_{n} \quad \forall n \leq m_{0} .
$$

Hence

$$
x_{m_{0}+1}>x_{m_{0}} \frac{\beta x_{m_{0}}}{1+\gamma x_{m_{0}-1}}>x_{m_{0}} \frac{\beta x_{m_{0}}}{1+\gamma x_{m_{0}}}>x_{m_{0}} \frac{\beta x_{0}}{1+\gamma x_{0}}>x_{m_{0}},
$$

i.e., $\left\{x_{n}\right\}$ is increasing. The condition $x_{0} \geq \bar{x}_{2}$ implies that $x_{n}$ tends to infinity.

(6) Suppose that $\beta>\gamma, \alpha>0$ and $1=4 \alpha(\beta-\gamma)$. Substituting by $\bar{x}=1 / 2(\beta-\gamma)$ in equation (2.3) one can easily see that $\bar{x}=1 / 2(\beta-\gamma)$ is unstable. The nonattractivity of $\bar{x}$ follows directly by considering a solution $\left\{x_{n}\right\}$ with the initial conditions $\left\{x_{-1}, x_{0}\right\}$ satisfying

$$
x_{0} \geq x_{-1} \text { and } x_{0} \geq \frac{1}{\beta-\gamma} \text {. }
$$

As in the proof of (5), it is easy to show that $\left\{x_{n}\right\}$ tends to infinity.

(7) Assume that $\beta>\gamma, \alpha>0$ and $1<4 \alpha(\beta-\gamma)$. Then in a similar way as in (5), one can easily show that the solution $\left\{x_{n}\right\}$ with the initial conditions $\left\{x_{-1}, x_{0}\right\}$ are such that

$$
x_{0} \geq x_{-1} \text { and } x_{0} \geq \frac{1}{\beta-\gamma}
$$


is increasing. Since equation (2.1) has no real equilibria, then $x_{n}$ tends to infinity.

3. THE EQUATION $x_{n+1}=x_{n}^{p} f\left(x_{n}, x_{n-1}, \ldots, x_{n-k}\right)$

Let $f \in C\left([0, \infty)^{k+1},(0, \infty)\right)$ such that $f$ satisfies the following conditions

(C1) $f\left(x, u_{1}, \ldots, u_{k}\right)$ is nonincreasing in $u_{1}, u_{2}, \ldots, u_{k}$.

(C2) $x^{p-1} f(x, x, \ldots, x)$ is increasing.

(C3) The equation $x^{p-1} f(x, x, \ldots, x)=1$ has a unique positive equilibrium $\bar{x}$.

We show that the asymptotic behaviour of the positive solutions of the difference equation

$$
x_{n+1}=x_{n}^{p} f\left(x_{n}, x_{n-1}, \ldots, x_{n-k}\right)
$$

depends on the initial conditions, see theorem 3.1. More precisely, we can choose the initial conditions such that the corresponding solution $\left\{x_{n}\right\}$ may tend to zero or infinity.

LEMMA 3.1. Assume that $\left\{x_{n}\right\}$ is a solution of equation (3.1). Under conditions (C1-C3) the following statements are true

(a) If for some $n_{0} \geq-k$,

$$
x_{n_{0}+k} \leq x_{n_{0}+\jmath}, j=0,1, \ldots, k-1 \text { and } x_{n_{0}+k}<\bar{x}
$$

then

$$
x_{n+k+1}<x_{n+k}<\bar{x} \forall n \geq n_{0} .
$$

(b) If for some $n_{0} \geq-k$,

$$
x_{n_{0}+k} \geq x_{n_{0}+\jmath}, j=0,1, \ldots, k-1 \text { and } \bar{x}<x_{n_{0}+k},
$$

then

$$
x_{n+k}<x_{n+k+1} \forall n \geq n_{0} .
$$

PROOF.

(a)Assume that for some $n_{0} \geq-k$,

$$
x_{n_{0}+k} \leq x_{n_{0}+\jmath}, j=0,1, \ldots, k-1 \text { and } x_{n_{0}+k}<\bar{x}
$$

Then

$$
\begin{aligned}
x_{n_{0}+k+1} & =x_{n_{0}+k}^{p} f\left(x_{n_{0}+k}, x_{n_{0}+k-1}, \ldots, x_{n_{0}}\right)=x_{n_{0}+k} x_{n_{0}+k}^{p-1} f\left(x_{n_{0}+k}, x_{n_{0}+k-1}, \ldots, x_{n_{0}}\right) \\
& \leq x_{n_{0}+k} x_{n_{0}+k}^{p-1} f\left(x_{n_{0}+k}, x_{n_{0}+k}, \ldots, x_{n_{0}+k}\right)<x_{n_{0}+k} .
\end{aligned}
$$

We can see by induction that

$$
x_{n+k+1}<x_{n+k}<\bar{x} \forall n \geq n_{0} .
$$

(b) Assume that for some $n_{0} \geq-k$,

$$
x_{n_{0}+k} \geq x_{n_{0}+\jmath}, j=0,1, \ldots, k-1 \text { and } \bar{x}<x_{n_{0}+k},
$$

Then

$$
\begin{aligned}
x_{n_{0}+k+1} & =x_{n_{0}+k}^{p} f\left(x_{n_{0}+k}, x_{n_{0}+k-1}, \ldots, x_{n_{0}}\right)=x_{n_{0}+k} x_{n_{0}+k}^{p-1} f\left(x_{n_{0}+k}, x_{n_{0}+k-1}, \ldots, x_{n_{0}}\right) \\
& \geq x_{n_{0}+k} x_{n_{0}+k}^{p-1} f\left(x_{n_{0}+k}, x_{n_{0}+k}, \ldots, x_{n_{0}+k}\right)>x_{n_{0}+k} .
\end{aligned}
$$


By induction we see that

$$
x_{n+k}<x_{n+k+1} \forall n \geq n_{0} .
$$

THEOREM 3.1. Under conditions (C1-C3) the following statements are true

If $\left\{x_{n}\right\}$ is a solution of equation (3.1) with initial conditions $\left\{x_{-k}, \ldots, x_{0}\right\}$ that satisfy

$$
x_{-\jmath} \geq x_{0}>0, j=1, \ldots, k \text { and } \bar{x}>x_{0},
$$

then $x_{n}$ tends to zero monotonically.

If the initial conditions $\left\{x_{-k}, \ldots, x_{0}\right\}$ are such that

$$
x_{-\jmath} \leq x_{0}, j=1, \ldots, k \text { and } \bar{x}<x_{0}
$$

then $x_{n}$ tends to infinity monotonically.

\section{PROOF.}

(1) From Lemma 3.1 we see that the solution $\left\{x_{n}\right\}$ is decreasing whence it converges to a nonnegative number, say $l$. Since $l<\bar{x}$, then $l=0$, because of condition (C3).

(2) We can see in a similar manner that $\left\{x_{n}\right\}$ is increasing and $x_{n}>\bar{x} \quad \forall n \in \mathbf{N}$. Therefore, $x_{n} \rightarrow \infty$ as $n \rightarrow \infty$ by condition (C3).

As a direct consequence we obtain the following result

COROLLARY 3.1. Under conditions (C1-C3), equation (3.1) is not permanent.

\section{MONOTONE SOLUTIONS OF $x_{n+1}=\beta x_{n}^{p} /\left(1+\sum_{i=1}^{k} \gamma_{i} x_{n-2}^{p-r}\right)$}

We apply theorem (3.1) to the rational recursive sequence

$$
x_{n+1}=\frac{\beta x_{n}^{p}}{1+\sum_{\imath=1}^{k} \gamma_{i} x_{n-\imath}^{p-r}},
$$

where $\beta>0, \gamma_{\imath} \geq 0 \forall i=1, \ldots, k, p \in\{2,3, \ldots\}, r \in\{1,2, \ldots, p-1\}$ and $\gamma=\sum_{\imath=1}^{k} \gamma_{\imath}>0$

We verify that the function $f\left(x, u_{1}, \ldots, u_{k}\right)=\beta /\left(1+\sum_{t=1}^{k} \gamma_{i} u_{i}^{p-r}\right)$ satisfies conditions (C1-C3). We can see easily that conditions (C1-C2) are satisfied. The equation

$$
x=\frac{\beta x^{p}}{1+\gamma x^{p-r}}
$$

has a unique positive solution if and only if the function

$$
h(x)=\beta x^{p-1}-\gamma x^{p-r}-1
$$

has a unique positive zero. Since

$$
h^{\prime}(x)=x^{p-r-1}\left[\beta(p-1) x^{r-1}-\gamma(p-r)\right],
$$

then we have the following two cases

If $r \in\{2, \ldots, p-1\}$, then $h$ has a unique positive zero $\bar{x}>[\gamma(p-r) / \beta(p-1)]^{1 / r-1}=x_{0}$ which is the unique equilibrium point of (4.1). Indeed, the function $h$ is decreasing for $0<x<x_{0}$ and increasing for $x>x_{0}$. Moreover, $\lim _{x \rightarrow \infty} h(x)=\infty$ and $h(0)=-1<0$. Then equation (4.1) has a unique positive equilibrium $\bar{x}$. 
If $r=1$ and $\beta>\gamma$, then equation (4.1) has the unique positive equilibrium

$$
\bar{x}=\left[\frac{1}{\beta-\gamma}\right]^{\frac{1}{p-1}} .
$$

Now we can apply theorem (3.1) to equation (4.1) to obtain the following result.

COROLLARY 4.1. Assume that either

$$
r \in\{2, \ldots, p-1\}
$$

or

$$
r=1 \text { and } \beta>\gamma \text {. }
$$

Let $\bar{x}$ be the unique positive equilibrium point of equation (4.1) and let $\left\{x_{n}\right\}$ be a solution of equation (4.1).

If for some $n_{0} \geq-k$

$$
x_{n_{0}+k} \leq x_{n_{0}+\jmath}, j=0,1, \ldots, k-1 \text { and } x_{n_{0}+k}<\bar{x}
$$

then

$$
x_{n+k+1}<x_{n+k} \forall n \geq n_{0} .
$$

If for some $n_{0} \geq-k$,

$$
x_{n_{0}+k} \geq x_{n_{0}+j}, j=0,1, \ldots, k-1 \text { and } \bar{x}<x_{n_{0}+k},
$$

then

$$
x_{n+k}<x_{n+k+1} \forall n \geq n_{0} .
$$

If the initial conditions $\left\{x_{-k}, \ldots, x_{0}\right\}$ are such that

$$
x_{-\jmath} \geq x_{0}>0, j=1, \ldots, k \text { and } \bar{x}>x_{0},
$$

then $x_{n}$ tends to zero monotonically.

If the initial conditions $\left\{x_{-k}, \ldots, x_{0}\right\}$ are such that

$$
x_{-\jmath} \leq x_{0}, j=1, \ldots, k \text { and } \bar{x}<x_{0}
$$

then $x_{n} \rightarrow \infty$ monotonically.

Now, we consider the equation

$$
x_{n+1}=\frac{\beta x_{n}^{p}}{1+\gamma x_{n-1}^{p-1}},
$$

where $\beta>0, \gamma>0, p \in\{2,3, \ldots\}$. We prove that there exists a solution $\left\{x_{n}\right\}$ which tends monotonically to $\bar{x}$. We follow the proof by Camouzis et. al. [2].

THEOREM 4.1. If $\beta>\max \{\gamma, 2 \sqrt{\gamma}\}$, then equation (4.2) has two solutions $\left\{x_{n}\right\}$ and $\left\{y_{n}\right\}$ such that $\left\{x_{n}\right\}$ increases to $\bar{x}$ and $\left\{y_{n}\right\}$ decreases to $\bar{x}$

PROOF. First, define the functions $f_{-1}$ and $f_{0}$ on $[0, \infty)$ by

$$
f_{-1}(x)=x^{2} \quad, \quad f_{0}(x)=x
$$


and

$$
f_{n+1}=\frac{\beta f_{n}^{p}}{1+\gamma f_{n-1}^{p-1}}, \quad n=0,1, \ldots
$$

Let

$$
A=\left\{x \in[0, \infty): \sup _{n \geq 0} f_{n}(x)<\bar{x}\right\}
$$

We show that $A \neq \emptyset$. Indeed, let $\theta$ be a positive number such that

$$
\theta<\min \left\{\bar{x},\left(\frac{\beta}{2 \gamma}-\frac{1}{2 \gamma} \sqrt{\beta^{2}-4 \gamma}\right)^{\frac{1}{p-1}}\right\}
$$

We have

$$
f_{1}(\theta)=\frac{\beta \theta^{p}}{1+\gamma \theta^{2 p-2}}
$$

One can easily show that $f_{1}(\theta)<f_{0}(\theta)=\theta<\bar{x}$. By Corollary $4.1(3), f_{n+1}(\theta)<f_{n}(\theta) \forall n \geq 0$. This implies that $\sup _{n \geq 0} f_{n}(\theta)=f_{0}(\theta)<\bar{x}$.

We define the function $S$ by

$$
S(x)=\sup _{n \geq 0} f_{n}(x)
$$

We claim that $S$ is continuous on $A$ and $A$ is open. Fix $x \in A$. There exists $N \geq 0$ such that

$$
f_{0}(x) \leq f_{1}(x) \leq \ldots \leq f_{N}(x)<\bar{x} \quad \text { and } \quad f_{N+1}(x)<f_{N}(x)
$$

If this were not true, then

$$
f_{0}(x) \leq f_{1}(x) \leq \ldots \leq S(x)<\bar{x},
$$

whence $f_{n}(x) \rightarrow S(x)=\bar{x}$ which is a contradiction. This implies that

$$
S(x)=f_{N}(x) \text { and } f_{N+1}(x)<f_{N}(x)
$$

Let $\epsilon>0$ be such that $\epsilon<\min \left\{\bar{x}-f_{N}(x),\left(f_{N}(x)-f_{N+1}(x)\right) / 2\right\}$. From the continuity of $f_{0}, \ldots, f_{N+1}$, there exists $\delta>0$ such that for $x^{\prime} \in A$ we have

$$
\left|x-x^{\prime}\right|<\delta \Rightarrow \sup _{0 \leq n \leq N+1}\left|f_{n}(x)-f_{n}\left(x^{\prime}\right)\right|<\epsilon
$$

Since $f_{N+1}\left(x^{\prime}\right)<f_{N+1}(x)+\epsilon<f_{N}(x)-\epsilon<f_{N}\left(x^{\prime}\right)<f_{N}(x)+\epsilon<\bar{x}$, then

$$
S(x)-\epsilon=f_{N}(x)-\epsilon<f_{N}\left(x^{\prime}\right) \leq S\left(x^{\prime}\right)
$$

and

$$
\begin{aligned}
S\left(x^{\prime}\right) & =\sup _{0 \leq n \leq N} f_{n}\left(x^{\prime}\right)<\sup _{0 \leq n \leq N}\left(f_{n}(x)+\epsilon\right) \\
& =f_{N}(x)+\epsilon<f_{N}(x)+\bar{x}-f_{N}(x)=\bar{x} .
\end{aligned}
$$

Therefore, $S$ is continuous and $A$ is open. Set $\lambda=\sup A$. Then $\lambda \notin A$ whence $S(\lambda) \geq \bar{x}$. The continuity of $f_{m}$ for every $m \geq 0$ implies that $S(\lambda) \leq \bar{x}$. Hence $S(\lambda)=\bar{x}$. Now, we claim that $f_{0}(\lambda)<f_{1}(\lambda)<\ldots<\bar{x}$. Indeed, we can see that $f_{1}(\lambda)>f_{0}(\lambda)$. If not, then $f_{0}(\lambda) \geq f_{1}(\lambda) \geq f_{2}(\lambda) \ldots$, because of corollary 4.1. Hence $S(\lambda)=f_{0}(\lambda)=\lambda=\bar{x}$ whence

$$
f_{1}(\lambda)=f_{1}(\bar{x})=\frac{\beta \bar{x}^{p}}{1+\gamma \bar{x}^{2 p-2}}=\frac{\beta(\bar{x})^{p}}{1+\gamma(\bar{x})^{p-1}} \frac{1+\gamma(\bar{x})^{p-1}}{1+\gamma \bar{x}^{2 p-2}}>\bar{x}
$$


Note that $\bar{x}<1$. Now assume that $f_{0}(\lambda)<f_{1}(\lambda)<\ldots<f_{N}(\lambda)$ and $f_{N}(\lambda) \geq f_{N+1}(\lambda)$ for some $N \geq 1$. Then $S(\lambda)=f_{N}(\lambda)=\bar{x}$ whence

$$
\begin{aligned}
f_{N+1}(\lambda) & =\frac{\beta f_{N}^{p}(\lambda)}{1+\gamma f_{N-1}^{p-1}(\lambda)}>\frac{\beta f_{N}^{p}(\lambda)}{1+\gamma f_{N}^{p-1}(\lambda)} \\
& =\frac{\beta \bar{x}^{p}}{1+\gamma \bar{x}^{p-1}(\lambda)}=\bar{x},
\end{aligned}
$$

which is a contradiction. Therefore, $f_{n}(\lambda)$ is increasing to $\bar{x}$.

Next, we define the functions $f_{-1}$ and $f_{0}$ on $[0, \infty)$ by

$$
f_{-1}(x)=x \quad, f_{0}(x)=x^{2}
$$

and

$$
f_{n+1}=\frac{\beta f_{n}^{p}}{1+\gamma f_{n-1}^{p-1}}, \quad n=0,1, \ldots
$$

We denote by

$$
A=\left\{x \in[0, \infty): \inf _{n \geq 0} f_{n}(x)>\bar{x}\right\} .
$$

We can see that $A \neq \emptyset$. Indeed, let $\theta$ be such that

$$
\theta>\max \left\{\sqrt{\bar{x}},\left(\frac{\gamma}{2 \beta}+\frac{1}{2 \beta} \sqrt{\gamma^{2}+4 \beta}\right)^{\frac{1}{p-1}}\right\} .
$$

We have

$$
f_{1}(\theta)=\frac{\beta f_{0}^{p}(\theta)}{1+\gamma f_{-1}^{p-1}(\theta)}=\frac{\beta \theta^{2 p}}{1+\gamma \theta^{p-1}}
$$

Set $a=\theta^{p-1}$. Then $a>\left(\gamma+\sqrt{\gamma^{2}+4 \beta}\right) / 2 \beta$ whence $\beta a^{2}>1+a \gamma$ i. e. $\left(\beta \theta^{2 p-2}\right) /\left(1+\gamma \theta^{p-1}\right)>1$. Hence $f_{1}(\theta)>f_{0}(\theta)=\theta^{2}>\bar{x}$. This implies that $\inf _{n \geq 0} f_{n}(\theta)=f_{0}(\theta)>\bar{x}$.

Define the function $S$ by

$$
S(x)=\inf _{n \geq 0} f_{n}(x) .
$$

We show that $S$ is continuous on $A$ and $A$ is open. In fact, fix $x \in A$, there exists a natural number $N$ such that

$$
f_{0}(x) \geq f_{1}(x) \geq \ldots \geq f_{N}(x)>\bar{x} \text { and } f_{N+1}(x)>f_{N}(x)
$$

Otherwise,

$$
f_{0}(x) \geq \ldots \geq f_{n}(x) \geq \ldots \geq S(x)>\bar{x}
$$

and therefore

$$
\lim _{n \rightarrow \infty} f_{n}(x) \geq S(x)>\bar{x}
$$

which is a contradiction. Hence

$$
S(x)=f_{N}(x) \text { and } f_{N+1}(x)>f_{N}(x) .
$$

Choose

$$
0<\epsilon<\min \left\{f_{N}(x)-\bar{x}, \frac{f_{N+1}(x)-f_{N}(x)}{2}\right\} .
$$


From the continuity of $f_{n}$, there exists $\delta>0$ such that

$$
\forall x^{\prime} \in[0, \infty)\left(\left|x-x^{\prime}\right|<\delta \Rightarrow\left|f_{n}(x)-f_{n}\left(x^{\prime}\right)\right|<\epsilon\right),
$$

where $n=0,1, \ldots, N+1$. Hence for $x^{\prime} \in(x-\delta, x+\delta) \cap[0, \infty)$ we have

$$
f_{N+1}\left(x^{\prime}\right)>f_{N+1}(x)-\epsilon>f_{N}(x)+\epsilon>f_{N}\left(x^{\prime}\right)>f_{N}(x)-\epsilon>\bar{x}
$$

Therefore

$$
S(x)+\epsilon=f_{N}(x)+\epsilon>f_{N}\left(x^{\prime}\right) \geq \inf _{n \geq 0} f_{n}\left(x^{\prime}\right)=S\left(x^{\prime}\right)
$$

Also

$$
S(x) \leq f_{n}(x)<f_{n}\left(x^{\prime}\right)+\epsilon \quad 0 \leq n \leq N
$$

Hence

$$
S\left(x^{\prime}\right)+\epsilon>S(x)
$$

and

$$
S(x)<S\left(x^{\prime}\right)+f_{N}(x)-\bar{x}
$$

This implies that $S\left(x^{\prime}\right)>\bar{x}$ and

$$
\left|S(x)-S\left(x^{\prime}\right)\right|<\epsilon,
$$

i.e., $S$ is continuous and $A$ is open.

Let $\lambda=\inf A$. Then $\lambda \notin A$. The continuity of $f_{n}$ for every $n$ implies that $S(\lambda)=\bar{x}$. Now, we show that $\left\{f_{n}(\lambda)\right\}_{n \geq 0}$ is decreasing to $\bar{x}$. We can see that $f_{1}(\lambda)<f_{0}(\lambda)$. Assume for the sake of contradiction that $f_{0}(\lambda) \leq f_{1}(\lambda)$. Then $\bar{x} \leq f_{0}(\lambda) \leq f_{1}(\lambda) \leq \ldots$ whence $S(\lambda)=f_{0}(\lambda)=\lambda^{2}=\bar{x}$. Hence

$$
f_{1}(\lambda)=\frac{\beta \lambda^{2 p}}{1+\gamma \lambda^{p-1}}=\frac{\beta \bar{x}^{p}}{1+\gamma \bar{x}^{\frac{p-1}{2}}}=\bar{x} \frac{1+\gamma \bar{x}^{p-1}}{1+\gamma \bar{x}^{\frac{p-1}{2}}}<\bar{x}
$$

which is a contradiction. By induction we can show that

$$
f_{0}(\lambda)>f_{1}(\lambda)>\ldots>\bar{x}
$$

\section{REFERENCES}

[1] E. CAMOUZIS, E. A. GROVE and G. LADAS, Monotone unstable solutions of difference equations and conditions for boundedness, J. Difference Equations and Appl. 1 (1995), 17-44.

[2] E. CAMOUZIS, G. LADAS, I. W. RODRIGUES and S. NORTHSHIELD, The rational recursive sequence $x_{n+1}=\left(\beta x_{n}^{2}\right) /\left(1+x_{n-1}^{2}\right)$, Computers Math. Applic. 28 (1994), 37-43.

[3] J. H. JAROMA, V. L. KOCIC and G. LADAS, Global asymptotic stability of a second-order difference equation, in J. Wiener and J. Hale, Eds, Partial Differential Equations, Pitman Research Notes in Mathematics, No. 273, Longman Scientific and Technical, Essex (1992) 80-84.

[4] V. L. KOCIC and G. LADAS, Permanence and global attractivity in nonlinear difference equations, Proceedings of the First World Congress of Nonlinear Analysis, Tampa, Florida, 1992.

[5] V. L. KOCIC and G. LADAS, Global behavior of nonlinear difference equations of higher order with applications, Kluwer Academic Publishers, Dordrecht, (1993).

[6] V. L. KOCIC, G. LADAS and I. W. RODRIGUES, On rational recursive sequences, J. Math. Anal. Appl. 173 (1993), 127-157. 


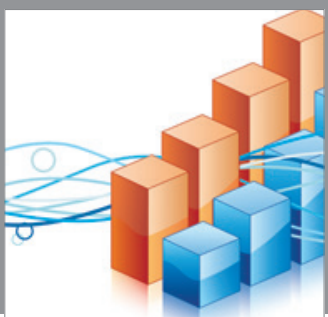

Advances in

Operations Research

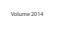

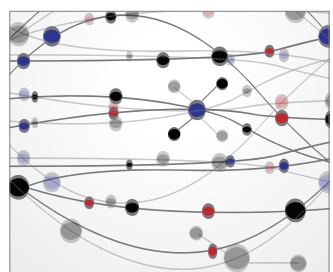

\section{The Scientific} World Journal
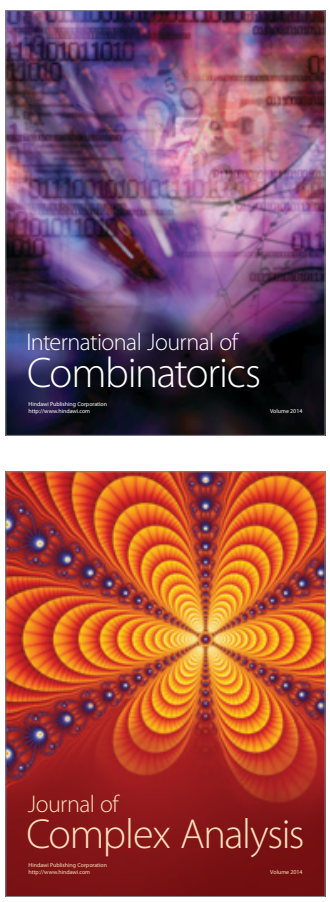

International Journal of

Mathematics and

Mathematical

Sciences
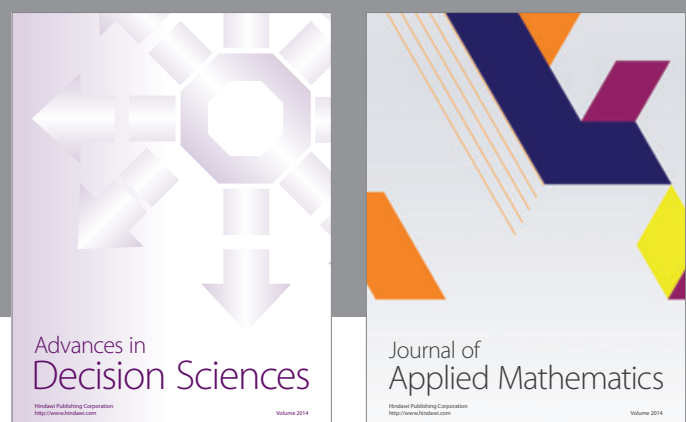

Journal of

Applied Mathematics
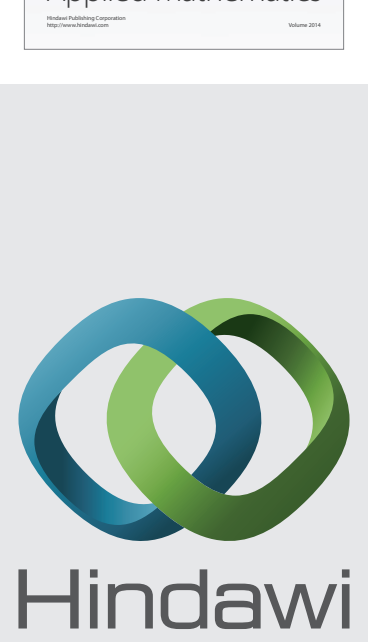

Submit your manuscripts at http://www.hindawi.com
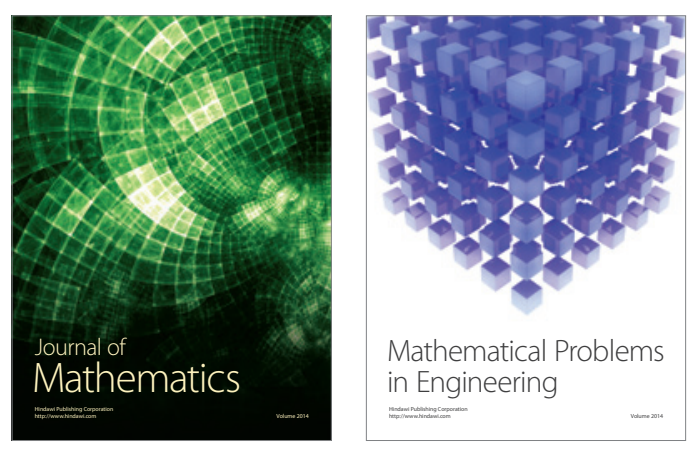

Mathematical Problems in Engineering
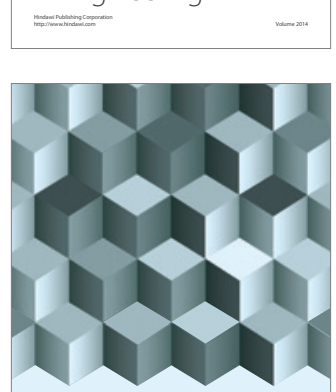

Journal of

Function Spaces
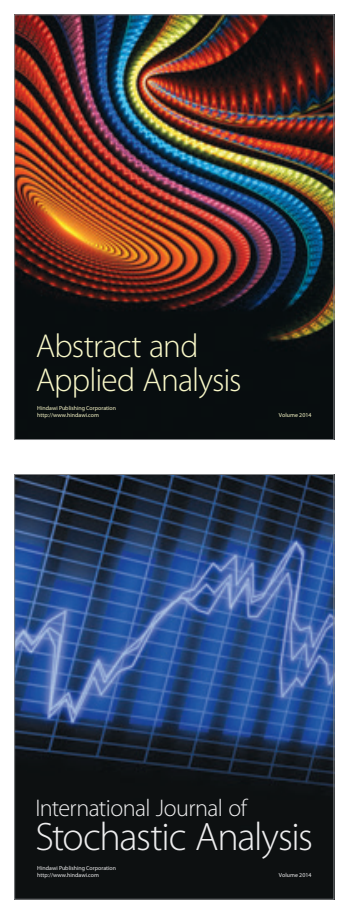

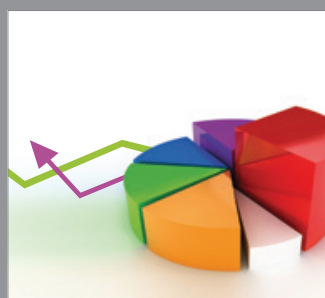

ournal of

Probability and Statistics

Promensencen
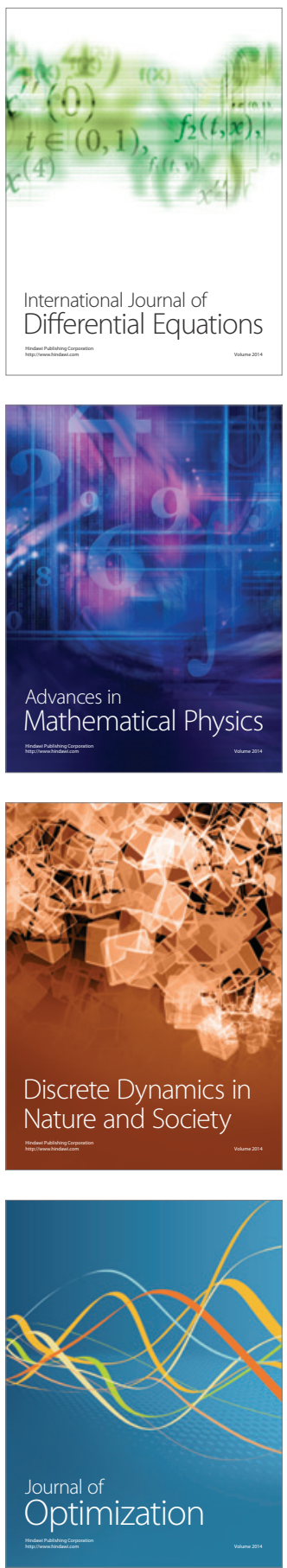